\title{
Autonomous Self-assembly in a Swarm-bot
}

\author{
Roderich Groß ${ }^{1}$, Michael Bonani ${ }^{2}$, Francesco Mondada $^{2}$, and Marco Dorigo ${ }^{1}$ \\ 1 IRIDIA, Université Libre de Bruxelles, Belgium \\ \{rgross,mdorigo\}@ulb.ac. be \\ 2 ASL, École Polytechnique Fédérale de Lausanne, Switzerland \\ $\{$ michael.bonani,francesco.mondada\}@epfl.ch
}

Summary. Multi-robot systems have been studied in tasks that require the robots to be physically linked. In such a configuration, a group of robots may navigate a terrain that proves too difficult for a single robot. On the contrary, many collective tasks can be accomplished more efficiently by a group of independent robots. This paper is about swarm-bot, a robotic system that can operate in both configurations and autonomously switch from one to the other.

We examine the performance of a single robot and of groups of robots selfassembling with an object or another robot. We assess the robustness of the system with respect to different types of rough terrain. Finally, we evaluate the performance of swarms of 16 physical robots.

At present, for self-assembly in autonomous, mobile robotics, swarm-bots is the state of the art for what concerns reliability, robustness and speed.

Key words: self-assembly, collective robotics, self-reconfigurable robotics, swarm robotics, swarm intelligence

\section{Introduction}

Many researchers aim at exploiting the potential of physically connected robotic modules to accomplish tasks that can not be achieved by the same modules separately. Groups of physically connected robots have been studied in the context of all-terrain navigation, for instance, navigating uneven terrain [13], descending stairs [23, 2], climbing near vertical walls [24], or avoiding obstacles during locomotion [21].

Special attention has been paid to the ability to self-reconfigure the shape, once connected into a common entity, with no external mechanical assistance $[8,3,22,19,24,14]$. In most of these systems, robotic modules either have to be pre-attached by the experimenter, or be arranged in specific relative positions. A single module usually has very limited autonomous capabilites. Typically it is not equipped with any sensors to perceive the environment (except simple IR photo sensors integrated in the connection plates). Nor, 
typically, are they capable of autonomous motion. Furthermore, the connection mechanism requires relatively accurate positioning (which is ensured, for instance, when operating within a grid-based configuration). These limitations, common to most self-reconfigurable robotic systems, make it difficult $[25,20,1]$, sometimes even impossible [2], to let two separate modules, or two groups of modules, connect autonomously.

We define a self-assembling system as a system composed of autonomous, mobile entities which can form artefacts of different size by physically connecting to and disconnecting from each other. To the best of our knowledge, there are only two multi-robot systems that are not subject to the limitations discussed above, and therefore capable of self-assembling.

- Fukuda et al. proposed the concept of dynamically reconfigurable robotic systems and realized an implementation with CEBOT, the first cellular robotic system $[5,8]$. CEBOT is a heterogenous system comprised of cells with different functions (e.g., move, bend, rotate, and slide). The autonomous docking of a single moving cell with an object cell has been demonstrated in an obstacle-free, flat terrain [7, 6]. Automatic assembly, disassembly and reconfiguration of up to three CEBOT cells have been realized by controlling a pair of conventional manipulators [9].

- Super Mechano Colony (SMC) is a new type of parent-child robot concept that has been proposed in the framework of Super Mechano Systems [12]. Two distinct systems have been developed: the first one consists of a passive mother-ship with attachable child robots, restricted to locomotion in 2D [4]. The ability of two child robots to detach from and to reconnect to the mother-ship has been demonstrated by making use of dead-reckoning. ${ }^{3}$ The second system, called SMC Rover, is a planetary rover with attachable child robots. As in the case of Millibot Trains [2], the current prototype lacks sensors to support autonomous docking. It has been developed to study the basic capabilities of the underlying mechanics [18].

We present a new robotic system called swarm-bot lying at the intersection between collective and self-reconfigurable robotics [16, see also http://www.swarm-bots.org]. The basic robotic entities, called s-bots, are fully autonomous and mobile. However, they can autonomously connect one to the other to form versatile structures that can self-reconfigure their shape.

\section{System Design}

\subsection{Hardware}

Fig. 1(a) shows the physical implementation of the s-bot. The robot has a height of $19 \mathrm{~cm}$ (including the transparent cylinder on top) and weighs approximately $700 \mathrm{~g}$.

\footnotetext{
${ }^{3}$ A video recording is available at http://www.ac.ctrl.titech.ac.jp/ yamakita/coe/smc.html.
} 


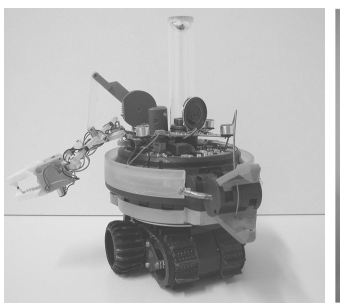

(a)

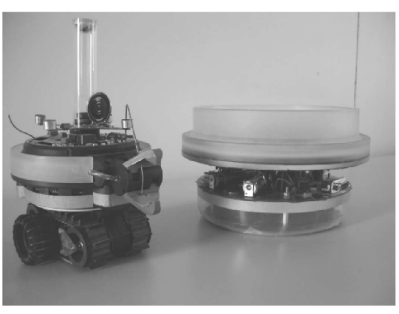

(b)

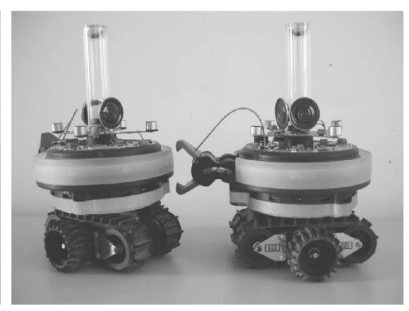

(c)

Fig. 1. (a) A single robot. Self-assembly with (a) an object, and (c) a teammate.

The s-bot has nine degrees of freedom (DOF) all of which are rotational, including two DOF for the traction system, one DOF to rotate the s-bot's upper part (called the turret) with respect to the lower part (called the chassis), one DOF for the grasping mechanism of the rigid gripper (in what we define to be the s-bot's front), and one DOF for elevating the arm to which the rigid gripper is attached (e.g., to lift another s-bot). A versatile arm attached to the side of the turret supports a second grasping device; the arm was not mounted when running the experiments presented in this paper. For the purpose of communication, the s-bot has been equipped with eight RGB LEDs distributed around the robot, and two loudspeakers.

The robot's traction system consists of a combination of tracks and two external wheels, called treels $\odot$. The tracks allow the s-bot to navigate on rough terrain. The diameter of the external wheels is slightly bigger than the one of the tracks which provides the s-bot with good steering abilities. To ensure a stable posture while enabling teammates to approach and connect from many different angles, the geometry of the treels@ has been chosen roughly cylindrical and of size comparable to the turret.

An s-bot can connect with another by grasping the connection ring. An s-bot can receive connections on more than two thirds of its perimeter. The mechanical design allows for some misalignment in all six DOF during the approach phase. A further fine-grained alignment occurs during the grasping phase. This is facilitated by the shape of the two teeth at the end of the gripper's jaws and by the relatively high force with which the gripper is closed $(15 \mathrm{~N})$.

The s-bot is equipped with a variety of sensors, including 15 proximity sensors distributed around the turret, four optical barriers integrated in the rigid and the semi-flexible gripper, and a VGA omni-directional camera. For a more comprehensive description of the s-bot's hardware see [17, 15].

\subsection{Controller}

We aim at controlling a group of s-bots in fully autonomous manner in such a way that they locate, approach and connect directly with an object that acts as a seed or with other $s$-bots already connected to the seed. 


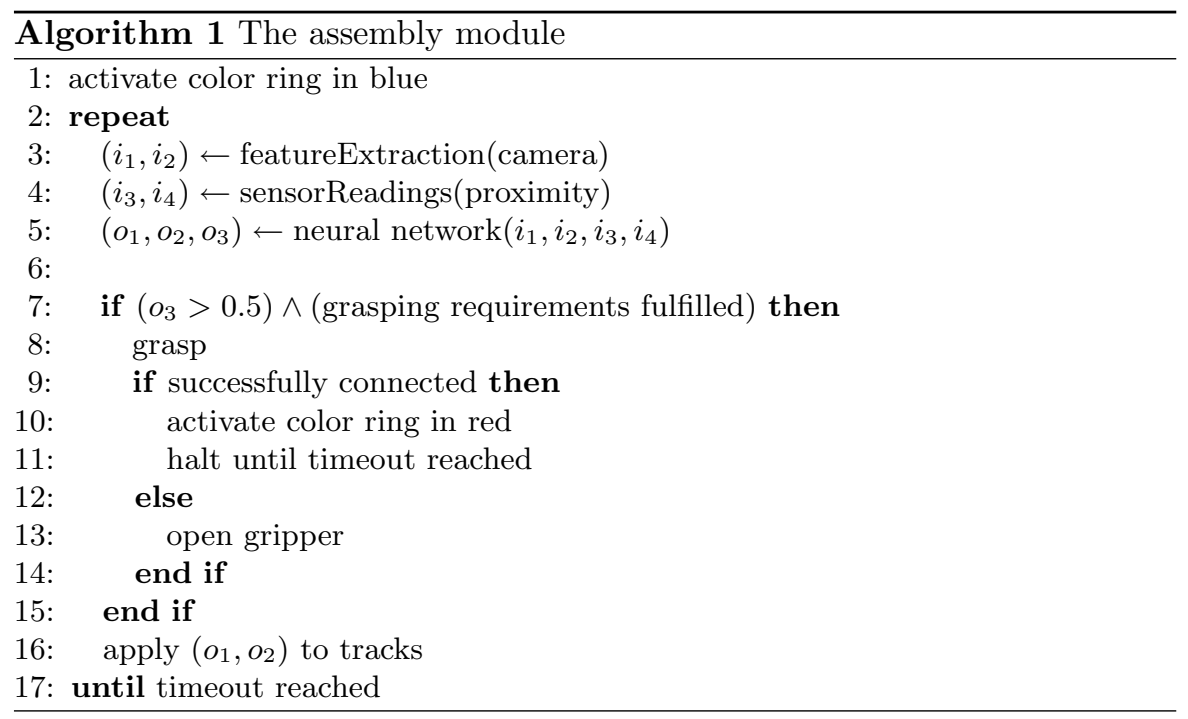

To favor scalability, the control is decentralized (i.e., fully distributed) and homogenous (i.e., all group members have identical control). The process of self-assembling is governed by the local attraction and repulsion among s-bots, and between s-bots and the seed. The color ring of the seed is permanently activated in red. Initially, all s-bots set the ring color to blue. The controller lets the s-bots avoid blue objects, and approach/connect with red objects. Thus, the process is triggered by the presence of the seed. Once an s-bot has established a connection, the color of its ring is set to red, attracting unconnected s-bots to connect with it. The basic principle of signaling the state (of being connected or unconnected) allows the emergence of (global) connection patterns of dimensions far beyond the robot's (local) sensing range.

Algorithm 1 details the control module for self-assembly. The main part is given by a reactive neural network (line 5 ) that maps sensory inputs to motor commands. The network takes as input the binary values $i_{1}$ and $i_{2}$ from the robot's vision system (line 3 ) and the values $i_{3}$ and $i_{4}$ from the left-front and right-front robot's proximity sensors (line 4$)$. The network's output $\left(o_{1}, o_{2}, o_{3}\right)$ is used to control the speed of the left and the right wheels (line 16) and the connection mechanism (lines 7 to 15$)$. By default, the tuple $\left(i_{1}, i_{2}\right)$ is assigned $(0,0)$. Any other assignment indicates the presence of red objects (in the front, or to the left or right side). If an obstacle (a blue object) is present in between, $i_{1}$ and $i_{2}$ are set to zero. The network's weights have been shaped by artificial evolution in the context of a cooperative transport task in simulation [11].

To avoid damage to the tracks and to improve the reliability of the control, we introduce a recovery move that is launched if high torque is continuously present on the tracks. During recovery, the s-bot moves about $5 \mathrm{~cm}$ backwards with a small lateral displacement. Afterwards the assembly module is resumed. 


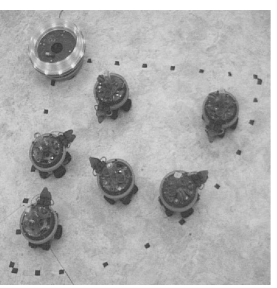

(a)

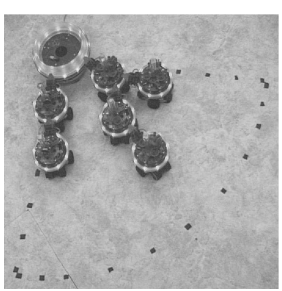

(b)

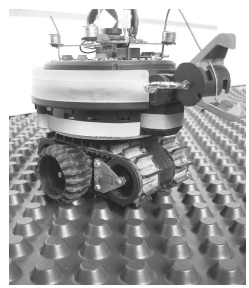

(c)

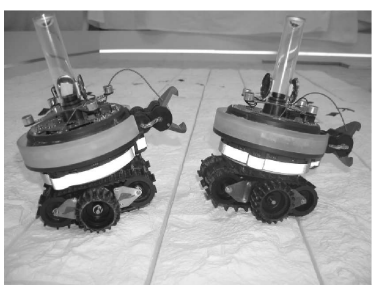

(d)

Fig. 2. Initial (a) and final (b) configuration of six s-bots self-assembling with the prey on flat terrain. Types of rough terrain: the moderately rough terrain (c), and the very rough terrain $(\mathrm{d})$.

\section{Results}

\subsection{Experiments on Flat Terrain}

We examine the ability of a single s-bot to approach and connect with an object, called prey (see Fig. 1(b)). The prey is equipped with a color ring of the same shape as the connection ring of the s-bots. The color is set to red. Initially, the s-bot is put at a specific distance $d \in\{25,50\}$ (in cm) and orientation $\alpha \in\left\{0^{\circ}, 90^{\circ}, 180^{\circ}, 270^{\circ}\right\}$ with respect to the prey. For each combination of $d$ and $\alpha$, five repetitions are carried out, thus in total 40 trials are performed. If the s-bot does not succeed in establishing a physical connection within $300 \mathrm{~s}$, the trial is stopped.

We repeated the experiment with four different s-bots. In all 160 trials, the s-bots succeeded in approaching and connecting with the prey. The average completion time for the 80 trials with distance $25 \mathrm{~cm}(50 \mathrm{~cm})$ is $22.6 \mathrm{~s}(34.9 \mathrm{~s})$.

We examine the ability of an s-bot to approach and connect to a (static) teammate (see Fig. 1(c)). We focus on the approaching angles $\alpha \in\left\{0^{\circ}, 60^{\circ}\right.$, $\left.120^{\circ}\right\}$, where $0^{\circ}$ corresponds to the target s-bot's tail. For each approaching angle, 20 trials have been performed. At the beginning of each trial, the approaching s-bot is put at a distance of $50 \mathrm{~cm}$ heading in the direction of its teammate. In all 60 trials, the s-bot did successfully connect. The average completion times for the 20 trials with approaching angle $0^{\circ}, 60^{\circ}$ and $120^{\circ}$ are $17.9,26.4$ and $17.9 \mathrm{~s}$, respectively.

So far, we have studied situations in which a single s-bot is approaching a single object for grasping. In the following, we assess the performance of a group of six s-bots accomplishing self-assembly with the prey given as an initial seed. Each s-bot is driven by identical control-the same as in the one-robot experiments.

At the beginning of each trial, the s-bots are positioned and oriented arbitrarily inside a circle of radius $70 \mathrm{~cm}$ around the prey. To favor potential interactions among the s-bots, we limited their initial positions to a $90^{\circ}$ segment of the circle. Fig. 2(a) and (b) show the initial and the final configuration in one typical trial. If the s-bot does not succeed within $600 \mathrm{~s}$, the trial is stopped. 
We performed 34 trials. In total, 199 times an s-bot succeeded in establishing a connection, while in only five occasions one s-bot failed. In 30 out of 34 trials, all seven objects were physically connected; on average this took $96 \mathrm{~s}$.

\subsection{Experiments on Rough Terrain}

We consider two types of rough terrain (see Fig. 2(c) and (d)). Both terrain types are unnavigable for most standard wheeled robots of a similar size. The first terrain type (here referred to as moderately rough terrain) has a surface with a regular structure. The second terrain type (here referred to as very rough terrain) consists of white plaster bricks providing a very rough, non-uniform surface.

We examine the ability of a single robot, and a group of six s-bot to approach and to establish a physical connection with the prey. Except for the difference in the terrain, the experimental set-up is identical to the one described in Section 3.1.

For each type of terrain, 40 trials have been performed with one s-bot and a prey. In each of the 40 trials on the moderately rough terrain a single s-bot successfully connected to the prey. On the very rough terrain, the s-bot failed only once for both initial distances. In the other 38 trials, the s-bot could successfully connect with the prey.

20 trials with groups of six s-bots have been performed on both types of rough terrain. For the moderately rough terrain, in total, 120 times an s-bot was controlled in order to establish a connection, and in 118 cases it could connect. For the very rough terrain we had to improve the control slightly, as it turned out to be too sensitive to the roughness. Using the modified controller, in 12 out of 20 trials all six s-bots connected with the prey. In total, 120 times an s-bot was controlled in order to establish a connection, and in 109 cases it succeeded.

\subsection{Experiments with 16 Physical Robots}

We examine the ability of a swarm of 16 s-bots to self-assemble without the presence of a prey. One s-bot acts as a seed, as it activates a pattern on its LED ring to attract teammates to approach from any direction but from the front (in which its connection mechanism does not allow for receiving connections).

The s-bot acting as a seed is put in the center of a circle of radius $50 \mathrm{~cm}$. Fifteen teammates are positioned and oriented arbitrarily within the same circle. The s-bots are positioned so that each s-bot can rotate on the spot without colliding with a teammate. The scenario is depicted in Fig. 3(a) and (b).

We repeated the experiment twelve times. In all but one case, all $16 \mathrm{~s}-$ bots successfully assembled to each other; on average this lasted $122 \mathrm{~s}$. In one case a single s-bot entered the connection state without being connected, and 


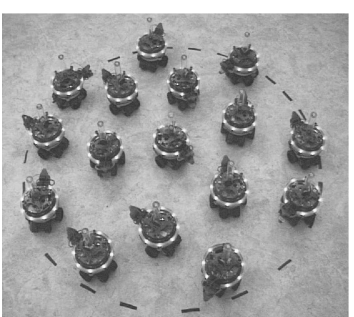

(a)

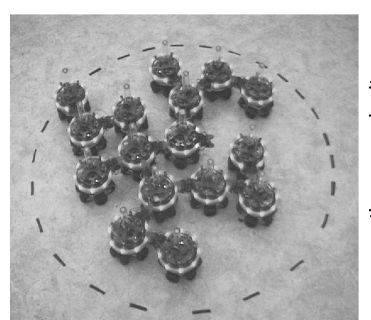

(b)

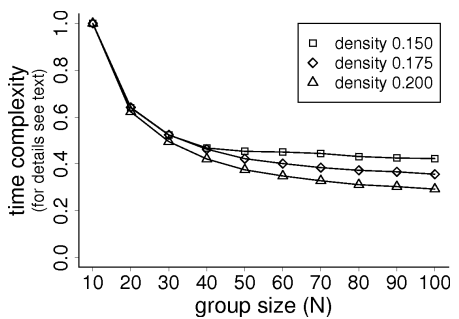

(c)

Fig. 3. Self-assembly in a robotic swarm. Initial (a) and final (b) configuration of 16 physical s-bots put in a circle of radius $50 \mathrm{~cm}$. Time complexity (c) for groups of 10 to 100 s-bots put in a circle around the prey (for different initial robot densities): average time (over 200 trials in simulation) it took an s-bot to connect, divided by the group size and scaled so that the performance for group size 10 equals 1 .

another s-bot connected with it; the other 14 s-bots connected with each other. Thus, in total, 190 out of 192 times an s-bot succeeded in task completion.

We studied self-assembly also in groups of 10 to 100 robots for different initial densities of robots. This study was carried out in simulation. We observed that the average time for a robot to connect scales sub-linearly with the group size (see Fig. 3(c) and [10] for more details).

\section{Conclusions}

In this article, we have presented a study of the problem of self-assembly in autonomous, mobile robots. At present, for the problem of self-assembly, swarm-bots is the state of the art for what concerns reliability and speed. Our system proved robust with respect to different initial conditions and different types of rough terrain. In addition, the system scales well with group size, as validated with 16 physical s-bots and up to 100 s-bots in simulation. The entire experimentation (i.e., 386 trials) was recorded on video (see http: //iridia.ulb.ac.be/ rgross).

Ongoing work indicates that by making use of the system's ability to selfassemble, we can address more complex robotic tasks, at the cutting edge of the current research in autonomous, collective mobile robotics.

Acting in between the two research fields of collective and self-reconfigurable robotics, we believe that the study of self-assembling robotic systems is a very promising direction for future research.

\section{Acknowledgments}

This work was supported by the "SWARM-BOTS Project", funded by the Future and Emerging Technologies programme (IST-FET) of the European 
Commission, under grant IST-2000-31010. The Swiss participants of the project are supported under grant 01.0012 by the Swiss Government. The information provided is the sole responsibility of the authors and does not reflect the Community's opinion. The Community is not responsible for any use that might be made of data appearing in this publication. Marco Dorigo acknowledges support from the Belgian FNRS, of which he is a Research Director, and from the "ANTS" project, an "Action de Recherche Concertée" funded by the Scientific Research Directorate of the French Community of Belgium. The authors want to thank all participants of the "SWARM-BOTS Project" for their great support. Additional thanks to Alexandre Campo and Davide Faconti for help in the development of the s-bot's vision system.

\section{References}

1. C. A. Bererton and P. K. Khosla. Towards a team of robots with repair capabilities: a visual docking system. In Proc. of the $7^{\text {th }}$ Int. Symp. on Experimental Robotics, volume 271 of LNCIS, pages 333-342. Springer, Berlin, Germany, 2000.

2. H. B. Brown, M.V. Weghe, C.A. Bererton, and P. K. Khosla. Millibot trains for enhanced mobility. IEEE/ASME Trans. Mechatron., 7(4):452-461, 2002.

3. A. Castano, W.-M. Shen, and P. M. Will. CONRO: Towards deployable robots with inter-robots metamorphic capabilities. Auton. Robots, 8(3):309-324, 2000.

4. R. Damoto, A. Kawakami, and S. Hirose. Study of super-mechano colony: concept and basic experimental set-up. Adv. Robots, 15(4):391-408, 2001.

5. T. Fukuda and S. Nakagawa. A dynamically reconfigurable robotic system (concept of a system and optimal configurations). In Proc. of the 1987 IEEE Int. Conf. on Industrial Electronics, Control and Instrumentation, pages 588595. IEEE Computer Society Press, Los Alamitos, CA, 1987.

6. T. Fukuda and S. Nakagawa. Method of autonomous approach, docking and detaching between cells for dynamically reconfigurable robotic system CEBOT. JSME Int. J. III-VIB. C., 33(2):263-268, 1990.

7. T. Fukuda, S. Nakagawa, Y. Kawauchi, and M. Buss. Self organizing robots based on cell structures - CEBOT. In Proc. of the 1988 IEEE/RSJ Int. Workshop on Intelligent Robots and Systems, pages 145-150. IEEE Computer Society Press, Los Alamitos, CA, 1988.

8. T. Fukuda and T. Ueyama. Cellular Robotics and Micro Robotic Systems. World Scientific Publishing, London, UK, 1994.

9. T. Fukuda, G. Xue, F. Arai, H. Asama, H. Omori, I. Endo, and H. Kaetsu. A study on dynamically reconfigurable robotic systems - assembling, disassembling and reconfiguration of cellular manipulator by cooperation of two robot manipulators. In Proc. of the 1991 IEEE/RSJ Int. Workshop on Intelligent Robots and Systems, volume 3, pages 1184-1189. IEEE Computer Society Press, Los Alamitos, CA, 1991.

10. R. Groß, M. Bonani, F. Mondada, and M. Dorigo. Autonomous self-assembly in mobile robotics. Technical Report IRIDIA/2005-2, IRIDIA - Université Libre de Bruxelles, 2005.

11. R. Groß and M. Dorigo. Group transport of an object to a target that only some group members may sense. In Proc. of the $8^{\text {th }}$ Int. Conf. on Parallel Problem 
Solving from Nature, volume 3242 of $L N C S$, pages 852-861. Springer Verlag, Berlin, Germany, 2004.

12. S. Hirose. Super mechano-system: New perspective for versatile robotic system. In Proc. of the $7^{\text {th }}$ Int. Symp. on Experimental Robotics, volume 271 of LNCIS, pages 249-258. Springer, Berlin, Germany, 2001.

13. S. Hirose, T. Shirasu, and E. F. Fukushima. Proposal for cooperative robot "Gunryu" composed of autonomous segments. Robot. Auton. Syst., 17:107-118, 1996.

14. M. W. Jørgensen, E. H. Østergaard, and H. H. Lund. Modular ATRON: Modules for a self-reconfigurable robot. In Proc. of the 2004 IEEE/RSJ Int. Conf. on Intelligent Robots and Systems, pages 2068-2073. IEEE Computer Society Press, Los Alamitos, CA, 2004.

15. F. Mondada, M. Bonani, S. Magnenat, A. Guignard, and D. Floreano. Physical connections and cooperation in swarm robotics. In Proc. of the $8^{\text {th }}$ Conf. on Intelligent Autonomous Systems, pages 53-60. IOS Press, Amsterdam, The Netherlands, 2004.

16. F. Mondada, L. M. Gambardella, D. Floreano, S. Nolfi, J.-L. Deneubourg, and M. Dorigo. The cooperation of swarm-bots: Physical interactions in collective robotics. IEEE Robot. Autom. Mag., 12(2):21-28, June 2005.

17. F. Mondada, G. C. Pettinaro, A. Guignard, I. W. Kwee, D. Floreano, J.-L. Deneubourg, S. Nolfi, L. M. Gambardella, and M. Dorigo. Swarm-Bot: A new distributed robotic concept. Auton. Robots, 17(2-3):193-221, 2004.

18. K. Motomura, A. Kawakami, and S. Hirose. Development of arm equipped single wheel rover: Effective arm-posture-based steering method. Auton. Robots, 18(2):215-229, 2005.

19. S. Murata, E. Yoshida, A. Kamimura, H. Kurokawa, K. Tomita, and S. Kokaji. M-TRAN: Self-reconfigurable modular robotic system. IEEE/ASME Trans. Mechatron., 7(4):431-441, 2002.

20. M. Rubenstein, K. Payne, P. Will, and W.-M. Shen. Docking among independent and autonomous CONRO self-reconfigurable robots. In Proc. of the 2004 IEEE Int. Conf. on Robotics and Automation, volume 3, pages 2877-2882. IEEE Computer Society Press, Los Alamitos, CA, 2004.

21. K. Støy, W.-M. Shen, and P. Will. On the use of sensors in self-reconfigurable robots. In Proc. of the $7^{\text {th }}$ Int. Conf. on Simulation of Adaptive Behavior, pages 48-57. MIT Press, Cambridge, MA, 2002.

22. K. Tomita, S. Murata, H. Kurokawa, E. Yoshida, and S. Kokaji. Self-assembly and self-repair method for a distributed mechanical system. IEEE Trans. Robot. Automat., 15(6):1035-1045, 1999.

23. M. Yim, D. G. Duff, and K. D. Roufas. PolyBot: a modular reconfigurable robot. In Proc. of the 2000 IEEE Int. Conf. on Robotics and Automation, volume 1, pages 514-520. IEEE Computer Society Press, Los Alamitos, CA, 2000.

24. M. Yim, K. Roufas, D. Duff, Y. Zhang, C. Eldershaw, and S. B. Homans. Modular reconfigurable robots in space applications. Auton. Robots, 14(2-3):225-237, 2003.

25. M. Yim, Y. Zhang, K. Roufas, D. Duff, and C. Eldershaw. Connecting and disconnecting for chain self-reconfiguration with PolyBot. IEEE/ASME Trans. Mechatron., 7(4):442-451, 2002. 